\title{
Deoxynivalenol and Nivalenol Toxicities in Cultured Cells: a Review of Comparative Studies
}

\author{
Hitoshi Nagashima \\ Food Research Institute, National Agriculture and Food Research Organization, 2-1-12 Kannondai, Tsukuba, \\ Ibaraki 305-8642, Japan
}

The in vitro studies of the toxicities of trichothecene mycotoxins deoxynivalenol (DON) and nivalenol (NIV) including cell proliferation, cytokine secretion, and the involvement of heat shock protein 90 (Hsp90) in their toxicities were reviewed. Trichothecene mycotoxins are extremely toxic to leukocytes and leukopenia is one of the leading signs of trichothecene toxicosis, implying that trichothecenes hinder cell proliferation. Both toxins retarded proliferation of all four cell lines tested. NIV was more potent than DON in human promyelocytic leukemia cell line HL60, human lymphoblastic leukemia cell line MOLT-4, and rat aortic myoblast cell line A-10. In contrast, both toxins exhibited almost the same potencies in human hepatoblastoma cell line HepG2. While exposure to $0.3 \mu \mathrm{g} / \mathrm{mL}$ DON greatly induced the secretion of anti-hematopoietic cytokines CCL3 and CCL4, treatment with NIV decreased the secretion of these cytokines in HL60 cells, indicating that the toxicity mechanisms of these mycotoxins differ. Because molecular chaperone Hsp90 occupies a pivotal position in a wide range of pathological processes, the effects of an Hsp90-specific inhibitor radicicol on cytokine secretions were investigated. Radicicol counteracted the effect of DON on cytokine secretion, indicating that Hsp90 plays a crucial role in DON-induced cytokine secretion in HL60 cells. Conversely, the results of co-treatment with NIV and radicicol indicate that radicicol does not mitigate the effect of NIV. Regarding CCL3 and CCL4 secretions, DON and NIV have Hsp90-related and -unrelated mechanisms of toxicities, respectively. Taken together the results suggest that, although these toxins share similar chemical structure, there are differences in their toxic mechanisms.

Key words: cell proliferation, cytokine secretion, deoxynivalenol, heat shock protein 90, nivalenol, radicicol

\section{Introduction}

Mycotoxins are secondary metabolites of various fungi and mycotoxin contamination of foodstuffs is a problem for many countries, particularly developing countries. For example, Fusarium fungi are commonly found on cereals grown in the temperate regions of the Americas, Europe, and Asia ${ }^{1}$. Diverse Fusarium species produce numerous mycotoxins of the trichothecene class and some other mycotoxins (e.g. zearalenone and fumonisins). More than 100 trichothecenes are now known; deoxynivalenol (DON, also called vomitoxin) and nivalenol (NIV) are two trichothecenes with highly similar chemical structures (Fig. 1). In Japan, NIV contamination as well as DON contamination of cereals is commonly found ${ }^{2,3)}$; hence, studies on both DON and NIV toxicities are needed. Since they occasionally cocontaminate Japanese wheat and barley ${ }^{2,3)}$, addressing the combined toxicity of these mycotoxins is also needed.

Group tolerable daily intake (TDI) is the superb concept for the governmental regulation of toxins, because the

Received: 26 October 2017; Accepted: 23 March 2018; Published online: 25 May 2018

Corresponding author: Hitoshi Nagashima, Ph.D. Food Research Institute, National Agriculture and Food Research Organization, 2-112 Kannondai, Tsukuba, Ibaraki 305-8642, Japan (nagasima@affrc.go.jp)

The contents of this article reflect solely the view of the author(s).

This paper was presented at the 12th International Symposium "Toxins, Pathogens, and Foods: Challenges and Opportunities for Public Health" organized by the Toxic Microorganisms Panel of the United States-Japan Cooperative Program on Development \& Utilization of Natural Resources.

Abbreviations: ANOVA: analysis of variance; DON: deoxynivalenol, Hsp90: heat shock protein 90, IARC: International Agency for Research on Cancer, $\mathrm{IC}_{50}: 50 \%$ inhibitory concentration, IL: interleukin, MIP: macrophage inflammatory protein, NIV: nivalenol, TDI: tolerable daily intake 


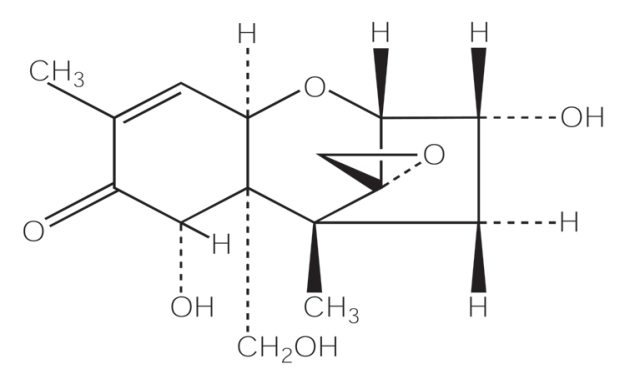

DON

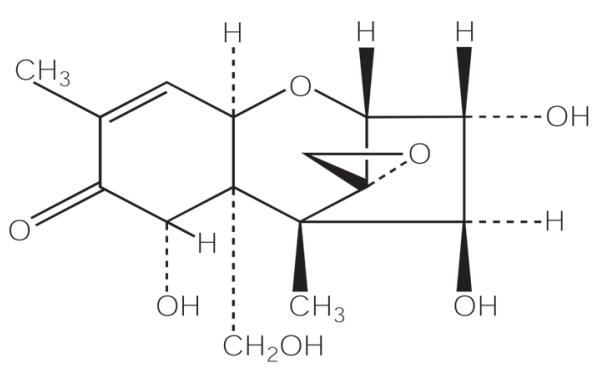

NIV

Fig. 1. Chemical structures of deoxynivalenol (DON) and nivalenol (NIV)

governments do not need to set the regulation values for each toxin. The prerequisite for establishing a group TDI is that no synergistic toxicity is observed, meaning that the mechanisms of toxicity are almost the same. In reality, Food Safety Commission of Japan, in addition to the European Commission Science Committee on Food, has a desire to set a group TDI for trichothecenes. Consideration has been suspended $^{4,5)}$ due to the insufficient evaluation of the toxicities of trichothecenes. Elucidation of detailed molecular mechanisms of trichothecenes' toxicities is strongly desired.

DON and NIV share highly similar chemical structures as the only difference between them is one oxygen atom at the 4 position in the trichothecene structure (Fig. 1). As is expected, they are reported to share many toxicological aspects such as the inhibition of cell proliferation ${ }^{6-8)}$, induction of CXCL8/interleukin (IL) -8 secretion $^{8,9)}$, and the involvement of stress-activated mitogen-activated protein kinases ${ }^{10,11)}$ and nuclear factor $\kappa \mathrm{B}^{12,13)}$ in the signal transduction pathways of toxicities. In addition, it is likely that neither DON nor NIV is carcinogenic. According to International Agency for Research on Cancer (IARC), they are classified as Group 3 (not classifiable as to its carcinogenicity to humans) ${ }^{14}$. The difference between carbon monoxide and carbon dioxide is the only one oxygen atom like the case of DON and NIV, nonetheless, toxicities of these carbon oxides are totally different. Therefore, to elucidate the differences between DON and NIV toxicities, I chose three indicators, cell proliferation, cytokine secretion, and the dependencies of 90-kDa heat shock protein (Hsp90) in toxicities, and investigated the differences in the response of these indicators to the two toxins.

\section{Cell Proliferation}

Trichothecene mycotoxins are extremely toxic to leukocytes, and alimentary toxic aleukia, a type of leukopenia, is one of the leading signs of trichothecene toxicosis ${ }^{15)}$, implying that trichothecenes hinder cell proliferation. Besides, cell proliferation is one of the most fundamental biological phenomena. I focused on the effects of DON and NIV on cell proliferation in various cultured cells and compared the potencies of both toxins ${ }^{16}$.

DON and NIV hindered cell proliferation in the human promyelocytic leukemia cell line HL60 and the 50\% inhibitory concentrations $\left(\mathrm{IC}_{50} \mathrm{~s}\right)$ were $0.36 \mu \mathrm{g} / \mathrm{mL}(1.22 \mu \mathrm{M})^{16)}$ and $0.16 \mu \mathrm{g} / \mathrm{mL}^{8)}$, respectively, indicating that DON is a less potent inhibitor of cell proliferation than NIV in this cell line (Fig. 2). Though the $\mathrm{IC}_{50}$ was slightly lower, the human lymphoblastic leukemia cell line MOLT-4 exhibited similar results (Fig. 2) which may be because both HL60 and MOLT-4 are leukemia cell lines. The rat aortic myoblast cell line A-10 showed the same trend as found in former two cell lines; that is, NIV was more potent than DON (Fig. 2). However, the $\mathrm{IC}_{50}$ of DON in A-10 cells was higher than that in the former two cell lines; consequently, the ratio of the $\mathrm{IC}_{50}$ of DON to that of NIV in A-10 cells was higher than that in the former two cell lines. The $\mathrm{IC}_{50}$ of DON in the human hepatoblastoma cell line HepG2 was similar to that in HL60 and MOLT-4 cells, while the $\mathrm{IC}_{50}$ of NIV was evidently higher than that in other cell lines and approximately equal to that of DON (Fig. 2). Contrary to the results from other cell lines, the potency of DON was the same as or even higher than that of NIV in HepG2 cells. With regard to cell proliferation, NIV is more potent inhibitor than DON in most of the cells tested ${ }^{6,7)}$, indicating that HepG2 is an exceptional case.

The ratios of the $\mathrm{IC}_{50}$ range from 0.9 (HepG2) to 5.3 (A10). Supposing that both DON and NIV exert their toxicities through the same mechanism but with a difference in potency, the ratios of the $\mathrm{IC}_{50}$ of DON to that of NIV are expected to be almost the same, regardless of the cell line. These results indicate the possibility that there are some differences in the mechanisms underlying the toxicities of DON and NIV. It should be noted, however, that other biological phenomena such as uptake, distribution, and metabolism of these two 


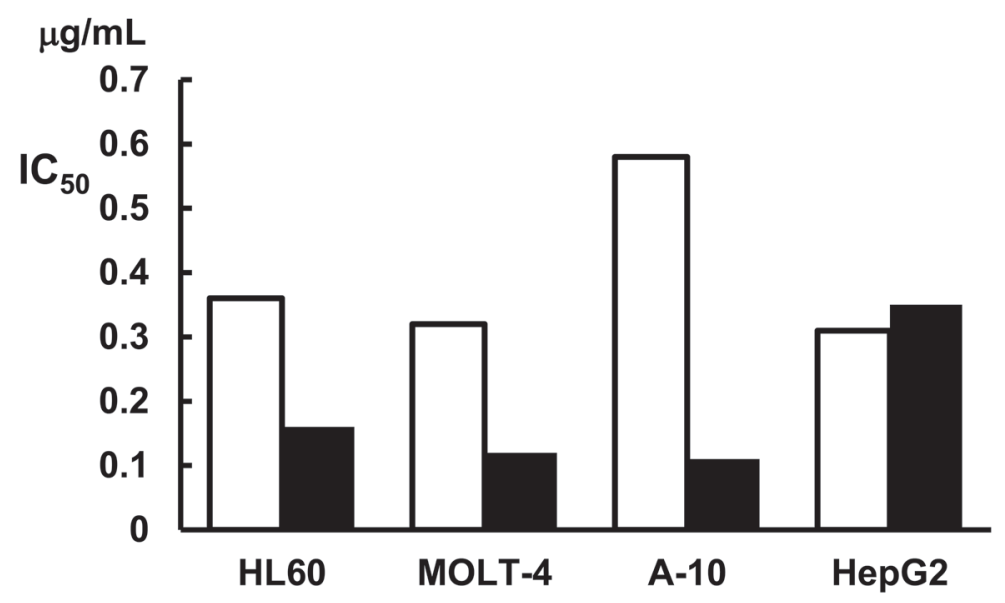

Fig. 2. Anti-proliferative effects of DON and NIV on HL60, MOLT-4, A-10, and HepG2 cells

Cells were treated with either DON or NIV for $24 \mathrm{~h}$. Open and filled bars represent treatment with DON and NIV, respectively. $\mathrm{IC}_{50}$ stands for $50 \%$ inhibitory concentration. Experiments were performed three times.

toxins also affect the results of cell proliferation. At present, the significance of the mechanisms of action on cell proliferation is uncertain, therefore, it should be elucidated in future studies.

The effects of T-2 toxin on cell proliferation were also documented for reference. T-2 toxin was much more potent than DON and NIV, and $\mathrm{IC}_{50}$ in A-10 cells and in HepG2 cells were $0.0055 \mu \mathrm{g} / \mathrm{mL}$ and $0.0054 \mu \mathrm{g} / \mathrm{mL}$, respectively. This tendency was consistent with the results of experiments using human lymphocytes ${ }^{17)}$.

\section{CCL3 and CCL4 secretion}

Cytokines are proteins secreted by various cell types, and each cytokine exerts a wide range of immune and inflammatory responses including anti-hematopoietic activity. Trichothecene mycotoxins induce leukopenia, presumably due in part to the inhibition of hematopoiesis. I addressed their effects on the secretion of anti-hematopoietic cytokines CCL3/macrophage inflammatory protein (MIP)-1 $\alpha$ and CCL4/MIP-1 $\beta^{18,19)}$ in HL60 cells ${ }^{20)}$.

Exposure to $0.3 \mu \mathrm{g} / \mathrm{mL}$ DON greatly $(P<0.05)$ and $1 \mu \mathrm{g} /$ $\mathrm{mL}$ DON moderately induced CCL3 secretion (Fig. 3A). Doses of $3 \mu \mathrm{g} / \mathrm{mL}$ or higher inhibited its secretion, presumably due to an adverse effect on the treated cells. DON affected the release of CCL4 in a similar manner as $0.3 \mu \mathrm{g} / \mathrm{mL}$ DON drastically induced CCL4 secretion $(P<0.05)$. Cells treated with $>1 \mu \mathrm{g} / \mathrm{mL}$ DON showed reduced CCL4 secretion compared with that in untreated cells (Fig. 4A). At 0.3 $\mu \mathrm{g} / \mathrm{mL}$ DON, the induction ratio of CCL4 was more marked than that of CCL3 (Fig. 3A, 4A). I showed that DON induces the secretion of the anti-hematopoietic cytokines CCL3 ${ }^{18,19)}$ (Fig. 3A) and CCL4 ${ }^{19)}$ (Fig. 4A). This indicates that DON has both direct anti-proliferative effects and indirect effects on the induction of anti-hematopoietic cytokine secretion and that these two effects may play important roles in the process of leukopenia.

Treatment with NIV reduced CCL3 secretion in a concentration-dependent manner (Fig. 3B). In comparison, low concentrations of NIV had modest inhibitory effects whereas high concentrations considerably inhibited CCL4 secretion (Fig. 4B). Because treatment with $10 \mu \mathrm{g} / \mathrm{mL}$ NIV was shown to cause widespread damage ${ }^{8)}$ in HL60 cells, NIV concentrations exceeding $10 \mu \mathrm{g} / \mathrm{mL}$ are unlikely to induce the secretion of cytokines. These results indicate that NIV, unlike DON, appears to induce neither CCL3 nor CCL4 secretion. These findings indicate that the mechanisms underlying the toxicities of DON and NIV evidently differ. That is, while DON induces the secretion of CCL3 and CCL4, NIV reduces their secretion.

\section{Involvement of Hsp90}

Hsp90 is an evolutionarily conserved molecular chaperone involved in the folding, stabilization, activation, and assembly of its client proteins. Numerous client signaling proteins involved in a wide range of biological processes have been found to be regulated by Hsp90 under physiological and pathological conditions ${ }^{21)}$. I focused on Hsp90 and investigated the effects of radicicol $^{22}$, an Hsp90-specific inhibitor, on cytokine secretion to elucidate whether Hsp90 is involved in the signal transduction pathway(s) of DON and NIV in 


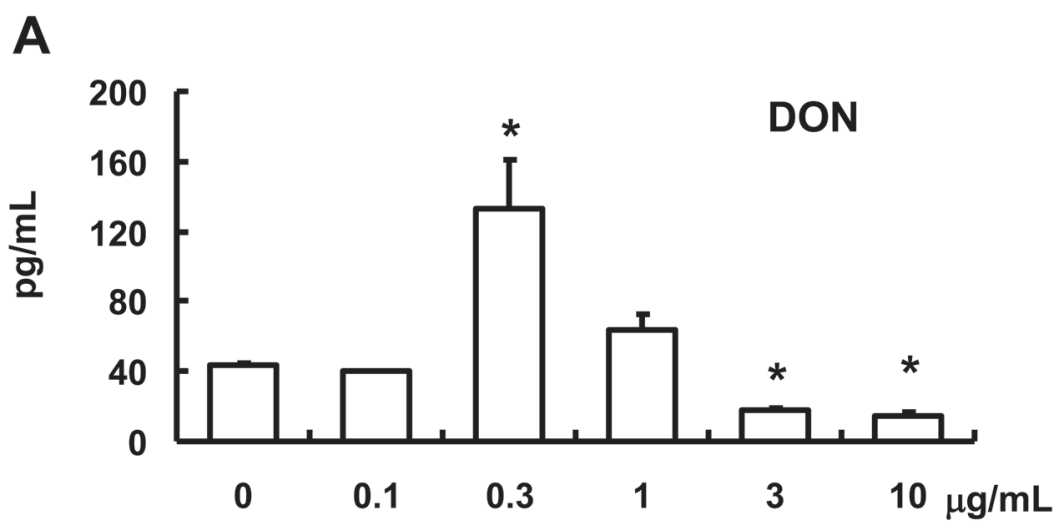

B

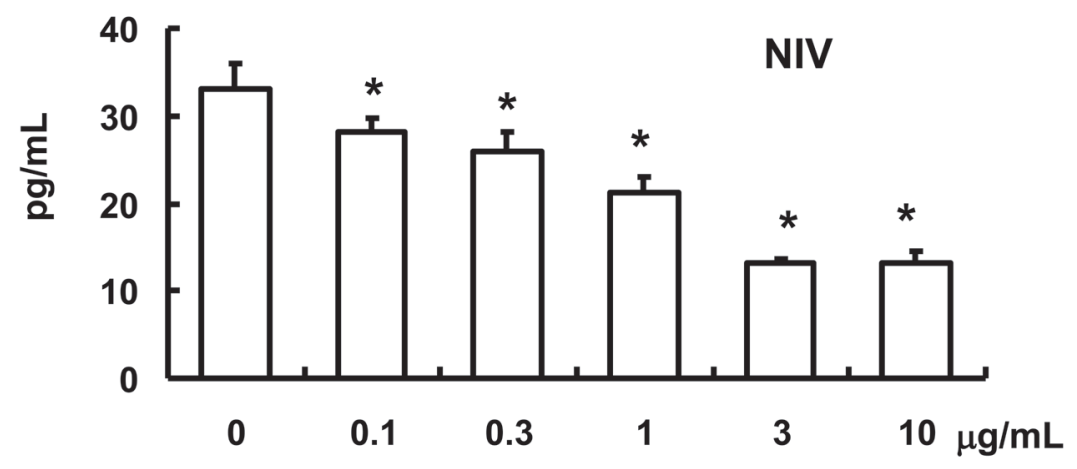

Fig. 3. Effects of DON (A) and NIV (B) treatments on CCL3 secretion in HL60 cells Cells were treated with either DON or NIV at the indicated concentrations for $24 \mathrm{~h}$. Values represent means \pm standard deviation $(\mathrm{n}=4)$. ${ }^{*}, P<0.05$ versus control dose $(0$ $\mu \mathrm{g} / \mathrm{mL}$ ). CCL3 was analyzed five times each using independent preparations.

\section{HL60 cells.}

DON significantly elicited CCL3 secretion (445.8\% of the value in the vehicle-treated samples). Conversely, radicicol alone decreased secretion (68.3\%; Table 1). The secretion of CCL3 in cells concomitantly treated with DON and radicicol was much lower $(73.6 \%)$ than that in cells treated with DON alone, and almost the same as treatment with radicicol alone (Table 1). DON significantly induced CCL4 secretion $(587.9 \%)$ and, similarly, radicicol alone slightly increased secretion (137.5\%; Table 1). The secretion of CCL4 in cells concomitantly treated with DON and radicicol was much lower $(151.9 \%)$ than that in cells treated with DON alone, and similar to the value of treatment with radicicol alone (Table 1). These results indicate that radicicol abolished the effects of DON, and that Hsp90 occupies an important position in DON-induced CCL3 and CCL4 secretion in HL60 cells $^{23)}$.

NIV moderately reduced CCL3 secretion $(84.7 \%$ of the value in the vehicle-treated samples) and radicicol alone halved secretion (52.6\%; Table 2). The secretion of CCL3 in cells concomitantly treated with NIV and radicicol was lower (48.6\%) than that in cells treated with NIV alone, and the same as treatment with radicicol alone (Table 2). Twoway analysis of variance (ANOVA) with replication does not show a statistically significant $(P<0.05)$ interaction between the effects of NIV and radicicol, meaning that the effects of NIV and radicicol are additive and Hsp90 is unlikely to be involved in NIV-caused reduction in CCL3 secretion in HL60 cells ${ }^{23}$. NIV reduced CCL4 secretion (82.4\%) while, conversely, radicicol considerably induced secretion (186.1\%; Table 2). The secretion of CCL4 in cells concomitantly treated with NIV and radicicol was lower $(70.9 \%)$ than that in cells treated with NIV alone, indicating that radicicol does not alleviate the effect of NIV ${ }^{23)}$ (Table 2). I previously reported that Hsp90 contributes to NIV-associated changes in CXCL8 and CCL2/monocyte chemotactic protein-1 secretion $^{24)}$ in HL60 cells, suggesting that there are at least two signal transduction pathways regarding the regulation of cytokine secretion. That is to say, Hsp90-dependent (CXCL8 and CCL2) and -independent (CCL3 and CCL4) pathways. I 

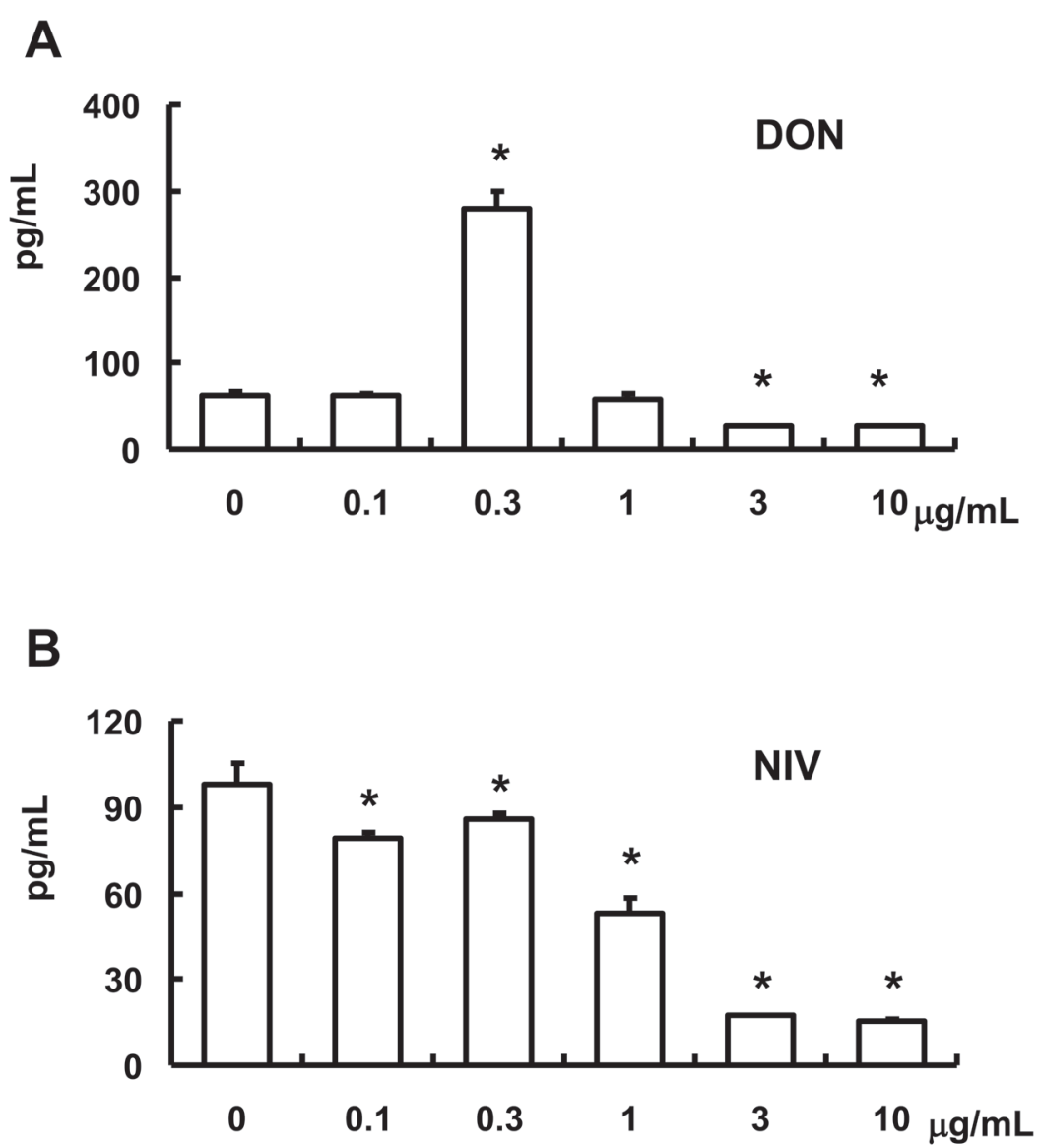

Fig. 4. Effects of DON (A) and NIV (B) treatments on CCL4 secretion in HL60 cells

Cells were treated with either DON or NIV at the indicated concentrations for 24 h. Values represent means \pm standard deviation $(\mathrm{n}=4) .{ }^{*}, P<0.05$ versus control dose $(0 \mu \mathrm{g} / \mathrm{mL})$. CCL4 was analyzed five times each using independent prepararion.

Table 1. The effects of radicicol on DON-induced changes in CCL3 and CCL4 secretion

\begin{tabular}{llcc}
\hline & & \multicolumn{2}{c}{$\operatorname{DON}(0.3 \mu \mathrm{g} / \mathrm{mL})$} \\
\cline { 3 - 4 } CCL3 & & - & + \\
& Vehicle & $100 \pm 3.0^{*}, *$ & $445.8 \pm 23.9^{*}, \S$ \\
& Radicicol $(1 \mu \mathrm{mol} / \mathrm{L})$ & $68.3 \pm 1.6^{*}$ & $73.6 \pm 1.3^{\S}$ \\
\hline \multirow{2}{*}{ CCL4 } & & & \\
& Vehicle & $100 \pm 9.8^{*}$ & $587.9 \pm 44.9^{*}, \S$ \\
& Radicicol $(1 \mu \mathrm{mol} / \mathrm{L})$ & $137.5 \pm 13.9$ & $151.9 \pm 6.4^{\S}$ \\
\hline
\end{tabular}

HL60 cells were treated with indicated chemicals for $24 \mathrm{~h}$. Results were expressed as mean \pm standard deviation $(n=4)$. CCL3 and CCL4 in the vehicle-treated samples were defined as $100 \%$. Intergroup differences were analyzed by Tukey's test. Intergroup differences between the values labeled with the same superscript symbols $(*,+$, and $\S)$ are statistically significant $(P<0.05)$. Experiments were performed four times. 
Table 2. The effects of radicicol on NIV-induced changes in CCL3 and CCL4 secretion

\begin{tabular}{|c|c|c|c|}
\hline & & \multicolumn{2}{|c|}{$\operatorname{NIV}(0.3 \mu \mathrm{g} / \mathrm{mL})$} \\
\hline & & - & + \\
\hline \multicolumn{4}{|l|}{ CCL3 } \\
\hline & Vehicle & $100 \pm 5.0^{*}$ & $84.7 \pm 10.3^{*}, \S$ \\
\hline & Radicicol $(1 \mu \mathrm{mol} / \mathrm{L})$ & $52.6 \pm 2.1$ & $48.6 \pm 1.9^{\S}$ \\
\hline \multicolumn{4}{|l|}{ CCL4 } \\
\hline & Vehicle & $100 \pm 7.6^{\ddagger}$ & $82.4 \pm 3.7$ \\
\hline & Radicicol $(1 \mu \mathrm{mol} / \mathrm{L})$ & $186.1 \pm 15.3^{\dagger, \dagger}$ & $70.9 \pm 3.4^{\dagger}$ \\
\hline \multicolumn{4}{|c|}{$\begin{array}{l}\text { HL60 cells were treated with indicated chemicals for } 24 \mathrm{~h} \text {. Results were expressed as } \\
\text { mean } \pm \text { standard deviation }(\mathrm{n}=4) \text {. CCL } 3 \text { and CCL4 in the vehicle-treated samples were } \\
\text { defined as } 100 \% \text {. Intergroup differences were analyzed by Tukey's test. Intergroup differ } \\
\text { ences between the values labeled with the same superscript symbols }(*, \dagger \text {, } \downarrow \text {, and } \S) \text { are } \\
\text { statistically significant }(P<0.05) \text {. Experiments were performed four times. }\end{array}$} \\
\hline
\end{tabular}

showed that with respect to CCL3 and CCL4 secretion, DON and NIV have Hsp90-related and -unrelated mechanisms of toxicities, respectively.

\section{Other Groups' results}

A number of groups have investigated DON and NIV toxicities, however, few groups reported on the evident differences between these two toxins ${ }^{9,25)}$. Sugita-Konishi and Pestka reported that DON induced tumor necrosis factor- $\alpha$ and IL-6 secretions in human macrophage-like cells, but NIV did not affect their secretion ${ }^{9)}$. Luongo et al. reported that NIV induced necrosis in murine dendritic cells, but necrosis was not observed in DON-treated cells ${ }^{25}$ ). On the other hand, the number of reports regarding synergisms between these two toxins is growing ${ }^{26-31)}$. These reports reinforce my idea that if two toxins exert their toxicities through the same mechanism, synergism of toxicities is not considered to be observed. Further studies are required, however, to identify which factor(s) contribute to difference(s) in the mechanisms of action of DON and NIV.

\section{Conflict of interest}

The author has no conflict of interest.

\section{References}

1. Creppy EE. Update of survey, regulation and toxic effects of mycotoxins in Europe. Toxicol Lett. 2002; 127: 19-28. PMID:12052637, doi:10.1016/S0378-4274(01)00479-9

2. Nakajima T, Yoshida M. Mycotoxin productivity and virulence of Fusarium graminearum species complex causing Fusarium head blight on wheat and barley in the western part of Japan. Japanese Journal of Phytopathology. 2007; 73: 106-111. doi:10.3186/jjphytopath.73.106

3. Yoshizawa T. Thirty-five years of research on deoxynivalenol, a trichothecene mycotoxin: with special reference to its discovery and co-occurrence with nivalenol in Japan. Food Safety. 2013; 1: 2013002. doi:10.14252/foodsafetyfscj.2013002

4. Scientific Committee on Food. Opinion of the Scientific Committee on Food on Fusarium toxins. Part 6: Group evaluation of T-2 toxin, HT-2 toxin, nivalenol and deoxynivalenol. European Commission, Brussel, Belgium; 2002.

5. Food Safety Commission of Japan. Risk assessment report: Deoxynivalenol and nivalenol (mycotoxin). Food Safety Commission of Japan, Tokyo, Japan; 2010.

6. Thuvander A, Wikman C, Gadhasson I. In vitro exposure of human lymphocytes to trichothecenes: individual variation in sensitivity and effects of combined exposure on lymphocyte function. Food Chem Toxicol. 1999; 37: 639-648. PMID:10478832, doi:10.1016/S0278-6915(99)00038-1

7. Minervini F, Fornelli F, Flynn KM. Toxicity and apoptosis induced by the mycotoxins nivalenol, deoxynivalenol and fumonisin B1 in a human erythroleukemia cell line. Toxicol In Vitro. 2004; 18: 21-28. PMID:14630058, doi:10.1016/S08872333(03)00130-9

8. Nagashima H, Nakagawa H, Iwashita K. Cytotoxic effects of nivalenol on HL60 cells. Mycotoxins. 2006; 56: 65-70.

9. Sugita-Konishi Y, Pestka JJ. Differential upregulation of TNF-alpha, IL-6, and IL-8 production by deoxynivalenol (vomitoxin) and other 8-ketotrichothecenes in a human macrophage model. J Toxicol Environ Health A. 2001; 64: 619636. PMID:11766169, doi:10.1080/152873901753246223 
10. Zhou HR, Islam Z, Pestka JJ. Rapid, sequential activation of mitogen-activated protein kinases and transcription factors precedes proinflammatory cytokine mRNA expression in spleens of mice exposed to the trichothecene vomitoxin. Toxicol Sci. 2003; 72: 130-142. PMID:12604842, doi:10.1093/ toxsci $/ \mathrm{kfg} 006$

11. Nagashima H, Nakagawa H, Kushiro M, Iwashita K. Contribution of stress-activated MAP kinases to nivalenol-caused cytotoxicity and interleukin-8 secretion in HL60 cells. JSM Mycotoxins. 2009; 59: 67-73.

12. Ouyang YL, Li S, Pestka JJ. Effects of vomitoxin (deoxynivalenol) on transcription factor NF- $\kappa \mathrm{B} / \mathrm{Rel}$ binding activity in murine EL-4 thymoma and primary $\mathrm{CD}^{+}{ }^{+} \mathrm{T}$ cells. Toxicol Appl Pharmacol. 1996; 140: 328-336. PMID:8887449, doi:10.1006/taap.1996.0228

13. Nagashima H, Kushiro M, Nakagawa H. Nuclear factor$\kappa \mathrm{B}$ inhibitors alleviate nivalenol-induced cytotoxicity in HL60 cells. Environ Toxicol Pharmacol. 2011; 31: 258-261. PMID:21787693, doi:10.1016/j.etap.2010.09.014

14. IARC Monographs on the Evaluation of Carcinogenic Risks to Humans, Vol. 56: Some naturally occurring substances: Food items and constituents, heterocyclic aromatic amines and mycotoxins. IARC, Lyon. 1993; 397-444.

15. Joffe AZ. Alimentary toxic aleukia. Microbial Toxins VII. Kadis S, et al, eds. New York, USA: Academic Press Inc; 1971: 139-189.

16. Nagashima H, Kushiro M, Nakagawa H, Iwashita K. Comparison of antiproliferative effects of trichothecene mycotoxins, nivalenol and deoxynivalenol, in cultured cells. Rep Nat'l Food Res Inst. 2012; 76: 29-32.

17. Johannisson A, Björkhag B, Hansson W, Gadhasson IL, Thuvander A. Effects of four trichothecene mycotoxins on activation marker expression and cell proliferation of human lymphocytes in culture. Cell Biol Toxicol. 1999; 15: 203-215. PMID:10696820, doi:10.1023/A:1007602011366

18. Graham GJ, Wright EG, Hewick R, et al. Identification and characterization of an inhibitor of haemopoietic stem cell proliferation. Nature. 1990; 344: 442-444. PMID:2320111, doi:10.1038/344442a 0

19. Graham GJ, Zhou L, Weatherbee JA, et al. Characterization of a receptor for macrophage inflammatory protein $1 \alpha$ and related proteins on human and murine cells. Cell Growth Differ. 1993; 4: 137-146. PMID:8385474

20. Nagashima H, Nakagawa H, Kushiro M. Opposite effects of two trichothecene mycotoxins, deoxynivalenol and nivalenol, on the levels of macrophage inflammatory protein (MIP)-1 $\alpha$ and MIP-1 $\beta$ in HL60 cells. Environ Toxicol Pharmacol. 2012; 34: 1014-1017. PMID:22964157, doi:10.1016/j. etap.2012.07.008
21. Pratt WB, Morishima Y, Osawa Y. The Hsp90 chaperone machinery regulates signaling by modulating ligand binding clefts. J Biol Chem. 2008; 283: 22885-22889. PMID:18515355, doi:10.1074/jbc.R800023200

22. Sharma SV, Agatsuma T, Nakano H. Targeting of the protein chaperone, HSP90, by the transformation suppressing agent, radicicol. Oncogene. 1998; 16: 2639-2645. PMID:9632140, doi:10.1038/sj.onc.1201790

23. Nagashima $H$, Nakagawa $H$. Differences in the toxicities of trichothecene mycotoxins, deoxynivalenol and nivalenol, in cultured cells. Japan Agricultural Research Quarterly: JARQ. 2014; 48: 393-397. .

24. Nagashima H, Nakagawa H, Kushiro M. Geldanamycin, an inhibitor of heat shock protein 90, mitigates nivalenol-caused changes in cytokine secretion in HL60 cells. Japan Agricultural Research Quarterly: JARQ. 2011; 45: 441-444. .

25. Luongo D, Severino L, Bergamo P, D’Arienzo R, Rossi M. Trichothecenes NIV and DON modulate the maturation of murine dendritic cells. Toxicon. 2010; 55: 73-80. PMID:19635492, doi:10.1016/j.toxicon.2009.06.039

26. Tajima O, Schoen ED, Feron VJ, Groten JP. Statistically designed experiments in a tiered approach to screen mixtures of Fusarium mycotoxins for possible interactions. Food Chem Toxicol. 2002; 40: 685-695. PMID:11955675, doi:10.1016/ S0278-6915(01)00124-7

27. Speijers GJA, Speijers MHM. Combined toxic effects of mycotoxins. Toxicol Lett. 2004; 153: 91-98. PMID:15342085, doi:10.1016/j.toxlet.2004.04.046

28. Alassane-Kpembi I, Kolf-Clauw M, Gauthier T, et al. New insights into mycotoxin mixtures: The toxicity of low doses of Type B trichothecenes on intestinal epithelial cells is synergistic. Toxicol Appl Pharmacol. 2013; 272: 191-198. PMID:23735874, doi:10.1016/j.taap.2013.05.023

29. Wan LYM, Woo CSJ, Turner PC, Wan JMF, El-Nezami H. Individual and combined effects of Fusarium toxins on the mRNA expression of pro-inflammatory cytokines in swine jejunal epithelial cells. Toxicol Lett. 2013; 220: 238-246. PMID:23688591, doi:10.1016/j.toxlet.2013.05.003

30. Cheat S, Pinton P, Cossalter AM, et al. The mycotoxins deoxynivalenol and nivalenol show in vivo synergism on jejunum enterocytes apoptosis. Food Chem Toxicol. 2016; 87: 45-54. PMID:26631294, doi:10.1016/j.fct.2015.11.019

31. Alassane-Kpembi I, Puel O, Pinton P, Cossalter AM, Chou TC, Oswald IP. Co-exposure to low doses of the food contaminants deoxynivalenol and nivalenol has a synergistic inflammatory effect on intestinal explants. Arch Toxicol. 2017; 91: 2677-2687. PMID:27915442, doi:10.1007/s00204-016-19029 